\title{
Ruptured Arteriovenous Malformation Presenting as Thalamic Hemorrhage
}

Santoshi Billakota ${ }^{1^{*}}$, Shahid M. Nimjee ${ }^{2}$ and Nada K. El Husseini $i^{1,3}$

${ }^{1}$ Department of Neurology, Duke University Medical Center, Durham, North Carolina, USA

${ }^{2}$ Department of Neurosurgery, The Ohio State University, Columbus, $\mathrm{OH}, \mathrm{USA}$

${ }^{3}$ Department of Neurology, Wake Forest Baptist Medical Center, Winston Salem, NC, USA

*Corresponding author: Santoshi Billakota, Box 3651, Duke University Medical Center, Durham, NC 27710, USA, E-mail: santoshi.billakota@duke.edu

Received date: July 10, 2015; Accepted date: July 27, 2015; Published date: July 292015

Copyright: (c) 2015 Billakota S. This is an open-access article distributed under the terms of the Creative Commons Attribution License, which permits unrestricted use, distribution, and reproduction in any medium, provided the original author and source are credited.

Keywords: Hypertensive hemorrhage; Thalamus; Arteriovenous malformation

\section{Introduction}

Brain Arterio Venous Malformations (AVMs) are congenital developmental vascular lesions consisting of direct arterial to venous connections without an intervening capillary network. AVMs are considered the most dangerous of the congenital vascular malformations with the potential to cause intracranial hemorrhage and seizures. The Spetzler-Martin grading system describes AVMs based on the size of the nidus, eloquence of the adjacent brain and venous drainage to give a score between 1 and 5 . The score correlates with morbidity and mortality after surgical resection. Grade 6 is used to describe inoperable lesions [1]. In a retrospective study analyzing the natural progression of AVMs in 343 patients, 90\% were found to be supratentorial involving the parietal lobe. Deep AVMs comprised $31 \%$ of all AVMs. Additionally, over $50 \%$ of patients were diagnosed before the age of 30 years with only $3 \%$ of AVMs diagnosed after the age of 60 years [2]. Because thalamic AVMs are not common and the thalamus is a common location for hypertensive hemorrhage, vascular imaging in subjects presenting with thalamic hemorrhage is not routinely performed.

We present a case of a 63-year-old man who presented with an extensive thalamic hemorrhage initially thought to be secondary to malignant hypertension. Digital subtraction arteriography (DSA) was performed after computerized tomography angiography (CTA) suggested vascular lesion, confirming a Spetzler Martin grade 3 thalamic AVM.

\section{Case Presentation}

A 63-year-old man with past medical history of hypertension and hyperlipidemia presented with acute onset of left sided weakness, blurred vision and headache. Non-contrasted CT brain showed a $2.7 \times$ $2.0 \mathrm{~cm}(17 \mathrm{cc})$ right thalamic hematoma with extensive IntraVentricular Hemorrhage (IVH) (Figure 1A). He was transferred to the intensive care unit (ICU) for further management. On exam, he was initially oriented to self and place and had mild dysarthria. Motor exam was notable for significant left sided weakness and brisk left sided reflexes. While in the ICU, he was transitioned from a nicardipine drip to three blood pressure agents (Metoprolol, Lisinopril and Hydralazine) to maintain systolic blood pressure (SBP) less than $140 \mathrm{mmHg}$. His ICU course was complicated by hydrocephalus due to intraventricular extension of blood and he had an external ventricular drain (EVD) placed and discontinued after a week. Upon transfer to the floor, a repeat CT scan showed a stable hemorrhage and blood pressure medications were progressively weaned. At that time, a CT angiogram (CTA) head was obtained and showed a right thalamic blush (Figure 1B). Digital subtraction angiogram confirmed a Spetzler Martin grade 3 right thalamic AVM (2 points for medium size of nidus and 1 point for deep venous drainage), supplied by branches of the right PCA, measuring approximately $2 \mathrm{~cm}$ in its widest diameter with early venous drainage into the vein of Galen (Figure 1C). Given location of the AVM, decision was made for future embolization and gamma knife radiation as opposed to resection. He was discharged to a nursing facility with plans to follow up in neurosurgery clinic in a few weeks.

\section{Discussion}

Intracranial AVMs are rare, with a prevalence of $15-18$ per 100,000 adults. Pooled data of 1860 reported AVM cases suggest that about $60 \%$ present with hemorrhage. The rate of hemorrhage in the course of untreated AVM is between $2 \%$ and $4 \%$ per year [2]. Anatomic and vascular features of the AVM also appear to influence risk of subsequent hemorrhage. These include the presence of associated aneurysms, deep venous drainage and deep brain location [3]. Our patient had the last two risk factors. As per the Columbia databank, AVMs which lacked hemorrhage at initial presentation, AVMs with deep brain location and deep venous drainage had an annual hemorrhage rate of 0.9 percent. In contrast, those with all three risk factors had an annual hemorrhage rate as high as 34.4 percent. Among 241 patients with untreated AVMs who initially presented with hemorrhage, a recurrent AVM hemorrhage occurred in 12 percent over an average 1.8 years of follow-up, yielding a 7 percent annual rebleeding rate [3].

Our case is interesting for two reasons. First, it is rare for cerebral AVMs to be diagnosed from a first time hemorrhage in patients over the age of 60 years. Second, the etiology of his hemorrhage was initially attributed to uncontrolled hypertension and the AVM could have been missed if a CTA had not been obtained. Hemorrhages produced by AVMs tend to be more often at the level of the cerebral convexity (subcortical white matter) than in the deep portions of the hemisphere, reflecting their usually more superficial location [4]. Nonhypertensive mechanisms account for as many as $55 \%$ of "lobar" ICH cases, while pontine hemorrhage is virtually always (90\%) hypertensive. Deep ganglionic (putaminal, thalamic) and cerebellar varieties occupy an intermediate position $(35 \%, 25 \%$, and $38 \%$ being of non-hypertensive mechanism, respectively) [4]. Although the lack of aggressive blood pressure control needed for the patient following the event may have indicated that his hemorrhage was not secondary to malignant hypertension, literature on this subject suggests that no sufficient method exists to differentiate intracranial hemorrhage (ICH) secondary to a structural cause (such as AVM) vs. uncontrolled hypertension. 
Citation: Billakota S, Nimjee SM, El Husseini NK (2015) Ruptured Arteriovenous Malformation Presenting as Thalamic Hemorrhage. J Cardiovasc Dis Diagn 3: 212. doi:10.4172/2329-9517.1000212
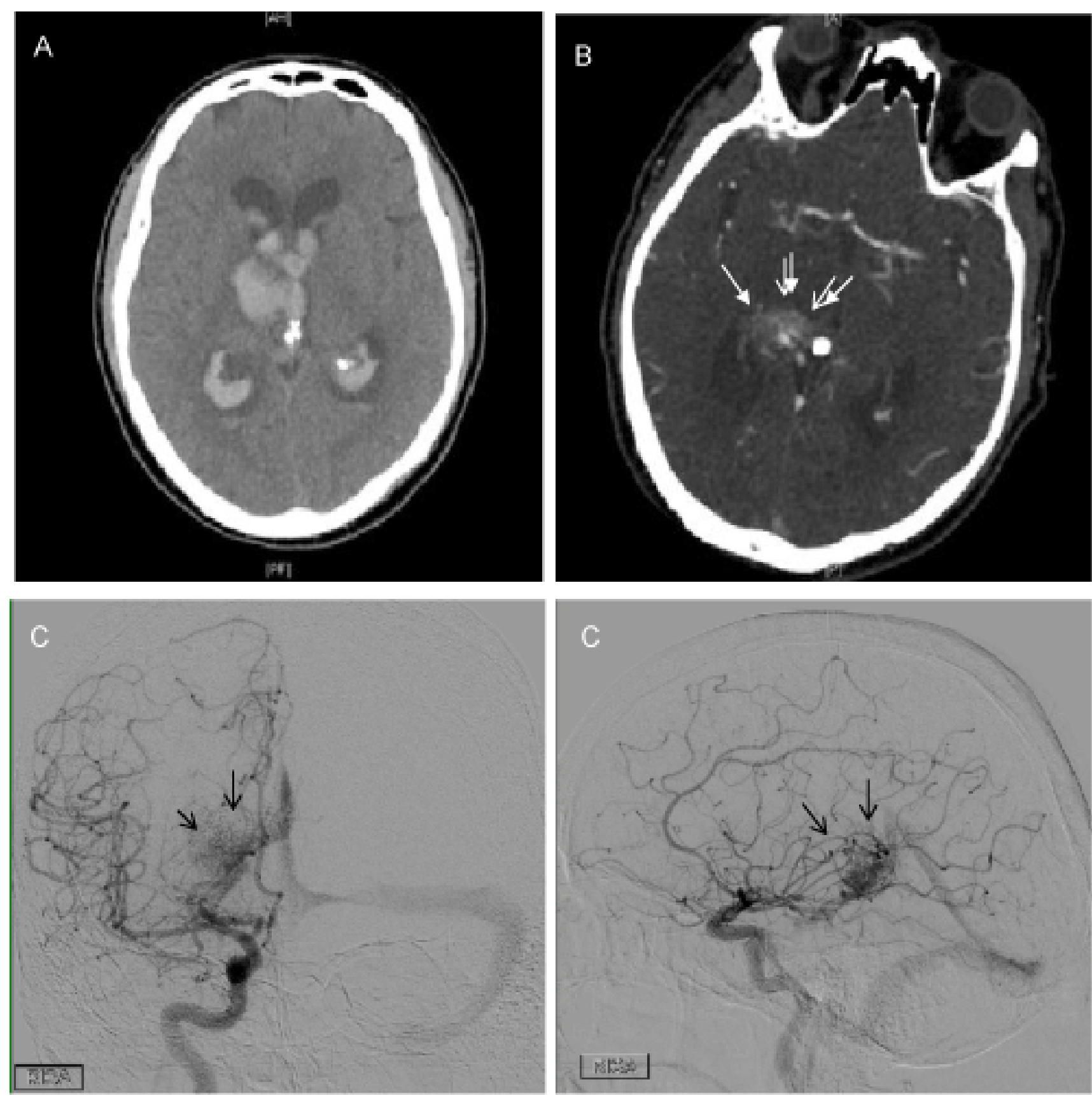

Figure 1: (A) Non-contrasted head CT showing thalamic hemorrhage and intraventricular blood; (B) CT angiogram with contrast demonstrating thalamic blush concerning for AVM; (C) Conventional arteriogram imaging showing AP and lateral imaging of thalamic AVM

The clinical exam and imaging (including location and intraventricular extension) could not have differentiated between the two. Hemorrhage secondary to AVMs present with ICH in any location, without specific clinical features other than perhaps a relatively slower course of development, as compared with the more rapidly evolving course of hypertensive ICH [5].

While AVM treatment is usually indicated for patients with a history of intracranial hemorrhage, treatment may not be indicated in the majority of patients with unruptured AVMs, particularly in the absence of other risk factors for hemorrhage. The Unruptured Brain Arteriovenous malformations (ARUBA) trial is the only randomized controlled trial that has studied treatment of brain AVMs [6]. Patients with unruptured AVMs were assigned to medical versus interventional (surgery, radiotherapy, and/or endovascular therapy) treatment. The trial was stopped early with outcome data on 223 patients followed for a mean 33 months. Composite rates of symptomatic stroke (ischemic 
Citation: Billakota S, Nimjee SM, El Husseini NK (2015) Ruptured Arteriovenous Malformation Presenting as Thalamic Hemorrhage. J Cardiovasc Dis Diagn 3: 212. doi:10.4172/2329-9517.1000212

Page 3 of 3

and hemorrhagic) and death were higher in the interventional compared to medical treatment group (31 versus 10 percent). Conservatively-treated patients had lower rates of neurological disability [6].

\section{Conclusion}

Even though uncommon, a thalamic hemorrhage with intraventricular extension can be the first presentation of AVM in patients over the age of 60 years. In the case presented, the hemorrhage was initially considered a hypertensive bleed until a CT angiogram was obtained. If vascular imaging had not been obtained, the AVM would have been missed.

\section{References}

1. Spetzler RF, Martin NA (1986) A proposed grading system for arteriovenous malformations. J Neurosurg 65: 476-483.
2. Crawford PM, West CR, Chadwick DW, Shaw MD (1986) Arteriovenous malformations of the brain: natural history in unoperated patients. Journal of Neurology Neurosurgery and Psychiatry 49: 1-10.

3. Mast H, Young WL, Koennecke HC, Sciacca RR, Osipov A, et al. (1997) Risk of spontaneous hemorrhage after diagnosis of cerebral arteriovenous malformation. The Lancet 350: 1065-1068

4. Stapf C, Mast H, Sciacca RR, Choi JH, Khaw AV, et al. (2006) Predictors of hemorrhage in patients with untreated brain arteriovenous malformation.Neurology 66: 1350-1355.

5. Kase CS (1986) Intracerebral hemorrhage: non-hypertensive causes. Stroke 17: 590-595.

6. Mohr JP, Parides MK, Stapf C, Moquete E, Moy CS, et al. (2014) Medical management with or without interventional therapy for unruptured brain arteriovenous malformations (ARUBA): a multicentre, nonblinded, randomised trial. Lancet 383: 614-621. 